\title{
La Semelfactivité non verbale en français : l'exemple de or et de (a)lors
}

\author{
Frédéric Torterat \\ Université de Nice - IUFM / EA 4080 \\ frederic.torterat@unice.fr
}

\section{Une semelfactivité non désignée comme telle}

Quelle place peut-on vraiment attribuer, dans le cadre des grammaires descriptives et des classements dictionnairiques, à ce qu'il est courant d'envisager comme les valeurs (variables) d'une conjonction ou d'un adverbe, ou encore ces cas particuliers qui semblent caractériser certains de leurs emplois ? Non pas qu'ils présentent forcément une certaine facilité, mais ces termes témoignent d'un embarras très concret du côté des rédacteurs, lesquels s'égarent quelquefois dans des intitulés passablement compliqués pour accompagner telle ou telle exemplification sur le plan historique. Ces hésitations justifient donc la présence de démarches complémentaires, pour ne pas dire inverses. C'est ainsi que, dans une autre problématique que celles de l'orcentricité et de la lorcentricité ou des valeurs, on peut analyser les modifications apparues dans les emplois de or et de (a)lors par l'intermédiaire de quelques invariants, et pour ainsi dire de ce qu'ils ont d'indéformable, comme c'est le cas pour la semelfactivité (Smith, 1991 ; Laca, 2002).

Dans une approche (qu'on peut appeler historico-comparative) dans laquelle nous nous plaçons ici, mais aussi en synchronie, la semelfactivité renvoie principalement à la catégorie verbale, notamment quand il est question de désigner une action « du point de vue de sa répétition » (Mantcheva 2004, 230) ou plus exactement comme «se produisant une fois » (Lefeuvre et Le Goffic 1999, 110. Cf. Dik, 1997 et Bres, 1999). Cela est aussi vrai dans le domaine du lexique français que dans celui, par exemple, des langues slaves, au titre desquelles les auteurs sollicitent volontiers cette problématique en termes d'Aktionsart (ainsi chez Stoll 1998, pour le russe). Pour autant, la semelfactivité conduit régulièrement les linguistes non seulement à généraliser leur réflexion sur les questions de la pluralisation et de la pluractionnalité, mais également à convenir du fait qu'elle concerne aussi bien la catégorie verbale que les déterminants nominaux (Buvet, Girardin, Gross et Groud, 2005), ainsi que d'autres éléments non verbaux, au premier rang desquels apparaissent les conjonctions et plus significativement les adverbes (Krifka et al., 1995 ; Benazzo, 2000 inter alii) ${ }^{1}$. Ces rapprochements sont d'autant plus productifs dans le cas d'emplois spécifiques de or et de (a)lors, pour ce qui nous occupe ici, où ces derniers marquent un instantané du discours soit présenté comme singulier, soit envisagé comme non répétitif, à ceci près que cet invariant des deux opérateurs n'est à proprement parler jamais vraiment présenté comme tel, mais décrit à l'appui d'un certain nombre de rôles qui sont autant de manières détournées de le conforter.

Ainsi Blumenthal, en 1980, dégagera-t-il le caractère "modificateur » de or, qui suggère « une idée de contraste, d'opposition » (132) entre l'arrière plan et le premier plan, l'événement nouveau et la situation générale. Une « valeur » à la fois temporelle et «transitive » sur laquelle revient Ruppli (1988, 403 sqq), et que Badiou-Monferran (2000, 242-255) scinde en opérations « temporelle » et « argumentative ». Dans d'autres termes, ceux de Bougy (2000), « le point commun [entre les significations relevées] est celui de la mise en évidence par or d'un élément de l'énonciation. Aussi, or serait un «maintenant» qui, en actualisant le procès qu'il introduit, en le mettant en évidence par rapport à ce qui précède, permettrait d'attirer l'attention de l'allocutaire / lecteur sur son importance » (62). Qui plus est, dans le cadre de ce rôle de «transition » que peuvent avoir co(n)textuellement or et (a)lors, il n'est pas toujours facile de distinguer leurs emplois (intra)diégétiques de ceux qui paraissent plus typiquement allocutifs - ou 
dialogaux, aussi les valeurs désignées se dispersent-elles dans une classification intermédiaire qui mérite pour le moins d'être réorganisée.

Concernant alors employé pour marquer l'instantanéité (singulative) en discours, une remarque d'Achard $(1992,587)$, partant de la problématique d'alors d'après son caractère hypothétiquement anaphorique ou déictique, établit une critique judicieuse du $T L F$, mais qui nous semble timide :

«Alors » ne peut donc pas s'interpréter comme anaphore, et son interprétation déictique, plus vraisemblable, n'est cependant pas satisfaisante. Son fonctionnement repose sur un rapport plus complexe : c'est la trace d'un opérateur ayant pour effet de clôturer le contexte temporel antérieur, en le renvoyant dans la situation ${ }^{2}$.

L'auteur livre pourtant ici des éléments de réponse assez facilement généralisables, en marge du fait que le terme de clôture paraisse moins approprié que celui de rupture, plus généralisable en français (AuthierRevuz, 1991 ; Ollier, 1995) - ce que d'ailleurs il nous confirme, quand il déclare, peu après, que alors rompt effectivement la continuité du discours, en indiquant, plus bas (588) :

Notre hypothèse rend compte sans forçage de l'emploi de "et alors? » en conversation. En effet, on peut considérer, avec C. Blanche Benvéniste et C. Jeanjean, qu'en conversation, c'est le même discours que partagent les interlocuteurs (...). «Et alors ?» renvoie donc à l'interlocuteur une situation dans laquelle ce qui vient d'être dit peut être considéré comme admis, mais où l'implication supposée n'est pas prise en charge. Le TLF classe en emploi temporel « et alors ? », et en emploi logique «ça alors! », « chic alors!» et «oh! si alors!». Notre analyse montre qu'il n'y a pas lieu de distinguer les deux emplois : « et alors?» prendra valeur temporelle par rapport à un récit, ou argumentative dans une discussion, mais sa valeur de trace d'opération reste la même. ${ }^{3}$

Si nous reprenons à ce titre Gerecht (1987), qui parle d'alors en termes d' "indicateur d'ancrage interpropositionnel ou interdiscursif» (69), nous nous voyons confirmer la manière dont d'aucuns envisagent d'ordinaire la capacité qu'a l'adverbe d'indiquer, par son instanciation même, un instantané discursif, à l'écart de toute forme d'itération, et plus ou moins ancré dans la sphère interlocutive.

Dans le même esprit, Jayez (1988) laisse de côté "certains emplois dialogaux et exclamatifs de alors » (133), mais dégage toutefois un emploi «temporel» et un emploi «énonciatif», parmi d'autres qu'il énumère en les exemplifiant en 134. L'auteur propose un inventaire de paraphrases qui témoignent de la polysémie cotextuelle de alors bien entendu, mais qui, également, nous invitent à envisager d'éventuelles coextensions de alors et d'autres opérateurs, comme ensuite et comme puis (135 sqq). A cette occasion, Jayez en vient à supposer l'existence de deux groupes d'emplois ( $T 1$ et $T 2)$. Pour ( $T 1)$ en effet, «les emplois temporels de alors ne sont pas forcément dominants et les autres emplois n'en sont pas dérivés », tandis que pour (T2), « le caractère temporel de alors est secondaire » (139). Or, il se reprend aussitôt :

T1 est à notre sens envisageable, mais T2 l'est beaucoup moins. T1 porte sur des emplois, alors que T2 porte sur un schéma temporel plus général qui serait censé rendre compte en tout ou partie des emplois, temporels ou non.

« Par commodité », indique-t-il (149), Jayez

distingu(e) un alors $_{1}$ et un alors 2 , bien que ces «deux » éléments aient des propriétés communes. Alors $s_{1}$ est celui qu'on trouve en tête ou en fin de phrase (avec détachement), et qu'on invoque souvent pour montrer qu'alors peut contrôler une énonciation; (ce marqueur) est le plus tolérant: il accepte l'utilisation phénoménale, inférencielle, et énonciative, seulement si les éléments forment une séquence $(\ldots)^{4}$. Alors $_{2}$ préfère l'utilisation phénoménale, aussi bien avec une séquence qu'avec un couple de phénomènes (partiellement) simultanés, tolère l'usage inférenciel, seulement en présence d'indices appropriés, et ne tolère jamais l'usage énonciatif (149-150; cf. 155, quand « la question ou l'impératif manipulent des contenus $p$ tels que : $p$ est (dans le contexte) une conséquence (déductive) modalisée du contenu du syntagme de gauche »).

Quoi qu'il en soit, on comprend, au milieu de ces paramètres, qu'il existe un emploi strictement temporel en lien plus ou moins direct avec un emploi inférenciel de l'opérateur (161 sqq), alors que le critère positionnel prend plus d'importance dans les démonstrations du linguiste au fur et à mesure qu'il s'efforce d'en désigner les «valeurs », dont celle qui consisterait à renvoyer à un simultané en discours (Cf. Franckel, 1989, 359, qui dégage aussi plusieurs de ces valeurs supposées en 364, et ses remarques 
sur la postposition en 366). Mais Jayez se refuse toutefois à rentrer dans des considérations en termes d'aspect non verbal, un terrain sur lequel, d'ailleurs, la plupart se résignent à ne pas se hasarder ${ }^{5}$.

\section{Quelles données sur le plan historique ?}

Dans le cadre dictionnairique, la répartition entre des emplois plutôt semelfactifs, ou plutôt itératifs de or et de (a)lors s'inscrit aussi dans un certain flottement. On notera pourtant que le TLF se dépêtre assez habilement des éventuelles ambivalences de or en assortissant la conjonction (en 2, le premier, 1, étant bien entendu l'homonyme substantif) de quelques cas particuliers, et or adverbe (3) de traits locutionnels. L'opérateur, comme conjonction de coordination, est d'abord envisagé «dans le récit», puis «dans l'argumentation», avant que certaines tournures spécifiques ne viennent compléter le classement (notamment dans un cadre argumentatif), là où l'adverbe est présenté comme archaïsant, et ensuite comme le noyau de locutions adverbiales (telles que d'ores en avant, d'ores et déjà) ou conjonctives (archaïsantes là encore, avec surtout ores que). En revanche, même si, dans l'historique dressé par le $T L F$, certains exemples se recoupent les uns les autres (comme la tournure en or del contralier!, en emploi « exclamatif», et or del bien querre (...), avec l'infinitif substantivé), l'ensemble de cet appendice constitue un relevé assez juste des emplois de or avant les années 1500. Ainsi sont répertoriées les occurrences de l'opérateur dans l'assertion (exclamative ou non) et la question, de même que dans l'injonction (avec, donc, or précédant l'infinitif substantivé). Concernant les emplois de l'adverbe dans la question notamment, le $T L F$ indique une «instance pressante ", avec un or au sens de donc, tout en reportant à la contribution de P. Imbs. D'autre part, un champ est laissé aux locutions interjectives ainsi qu'aux structures en or que : tous ces emplois sont distingués de ceux où or correspond à un caractère plus « argumentatif », dans la plupart des cas semelfactif, bien que l'on reconnaisse, dans certains d'entre eux, des variables des précédents. La présence d'un tel exposé dans l'article du TLF formalise néanmoins l'aveu que, malgré un éparpillement supposé des emplois de or avant les années 1500, l'accroissement de ceux de l'opérateur en tant que coordonnant s'est produit dans la suite prévisible d'un usage qui favorisera de plus en plus sa dimension de conjonction singulative, où l'on reconnaît souvent un caractère argumentatif sans rupture tranchée avec sa dimension temporelle. A ce sujet, une remarque d'Antoine (1962, 1195) mérite sans doute d'être rapportée, quoiqu'elle se retranche en partie, comme on peut s'y attendre, derrière le terme de valeur :

(...) c'est souvent à la faveur de ce passage d'un temps objectif à un temps subjectif que s'est produit - avec or comme avec d'autres termes (donc, si par exemple) - le glissement de la valeur temporelle à une valeur abstraite. Un cas particulièrement frappant, d'ailleurs beaucoup plus simple, de ce glissement, est celui du or qui, après digression, sert à reprendre le fil de la narration interrompue, ou qui, à la fin d'un développement, sert à introduire une formule de résumé.

C'est donc dans un instant textuel que plusieurs emplois de or se rassemblent opportunément : ici l'opérateur indique le moment singulier d'une digression, là celui d'une formule résomptive, et son rôle de cohéreur textuel s'appuie invariablement sur une semelfactivité de fait que le contexte verbal vient la plupart du temps corroborer. Mais chez Antoine comme chez ses prédécesseurs, la possibilité de dégager une vision continuiste est laissée en suspens (Feuillet, 1990), alors qu'en diachronie, cette semelfactivité s'avère pleinement opératoire, comme ici :

Mercure - Dictes-vous ? Ha ! vous en riez et vous en mocquez ? Non ! vous ne vivrez pas tant voirement, et si serez tout le temps de vostre vie en servitude et malade toutes les lunes jusques au sang. Or voy-je bien que la maulvaistié des femmes surmontera celle des hommes. (Cymbalum Mundi, I, Droz, p. 10)

Mercure - Non, non; je feray bien mieulx : je m'en voys changer mon visage en aultre forme. Or me regarde bien au visage, pour veoir que je deviendray. (Ibid., II : p. 14)

Concernant à nouveau le $T L F$, c'est donc dans l'homonyme adverbe, lequel bénéficie bien sûr d'une entrée spécifique, que or, employé ou non en locutions (et notamment une fois encore avec mais), rassemble des acceptions strictement temporelles, avec pour parasynonyme, entre autres, le terme présentement. Sont exposées ses graphies ore et ores, que le TLF déclare vieillies ou lexicalisées, 
conformément à ce qui en a été conclu précédemment (Cf. Badiou-Monferran, 2003). Nous indiquerons pourtant un fait non négligeable : l'ouvrage note toujours un «emploi interjectif, renforcé par ça, sus, pour interpeller, pour exciter, pour convier à faire qqc », ainsi que l'emploi de or avec un impératif semelfactif (or écoutez (...) ). Par ailleurs, les locutions en d'ores (...) et ores que sont elles aussi rappelées à titre particulier. Outre les extraits cités, qui forment un ensemble d'une certaine cohésion, c'est à l'historique lui-même, très fourni, que revient à notre sens le plus grand mérite de l'article. Car, bien que cet appoint prolonge à peu près la classification à laquelle il a été procédé au fil du classement des acceptions de l'opérateur en synchronie dans or conjonction (2), et or adverbe (3), le TLF confirme que l'adverbe de «l'articulation logique », attesté dès le Chevalier au Lion, et marquant ainsi « un point important dans l'enchaînement de la pensée, d'une phrase à une autre ", renvoie à un " emploi conduisant vers celui de conjonction de coordination », ce qui implique, évidemment, une distinction homonymique postérieure, dont il reste à problématiser le suivi.

La même question se pose néanmoins pour (a)lors qui, depuis ses premières apparitions dans les textes, intervient de manière récurrente dans le récit pour délimiter les moments de l'action narrative et, en particulier, pour démarquer les événements qu'il inscrit, sur un plan phrastique comme sur le plan textuel, dans un instant singulier ${ }^{6}$. Très présent dans les chroniques de Clari et de Froissart, où il demeure concurrencé par atant et einsi, il conserve une certaine consistance événementielle dès lors qu'il contribue à dramatiser un moment de la diégèse présenté comme déterminant, d'où sa présence comme opérateur semelfactif dans des constructions hypotaxiques, et notamment dans le récit :

$$
\begin{aligned}
& \text { Quant j'ay servi longuement } \\
& \text { Lors vient ingratitude et bruit; } \\
& \text { D'estat me depointe et me nuit. } \\
& \text { (Eustache Deschamps, ball. 1253, } 3^{\mathrm{e}} \text { strophe) }
\end{aligned}
$$

De tels emplois s'inscrivent dans une invariabilité pour ainsi dire facile à démontrer, qui plus est dans les configurations où le récit lui-même comprend des cas de concaténation, comme c'est le cas dans les deux extraits suivants, lesquels ne sont par ailleurs similaires qu'en partie :

Et lor pria que cele dame li gardassent laiens jusques a son revenir, et on li otria molt volentiers.

(Istore d'Outre Mer, in F. Godefroy, Dict.)

Elle ne dit rien d'autre, elle ne lui dit pas laissez-moi tranquille. Alors il a moins peur. Alors il lui dit qu'il croit rêver. Elle ne répond pas. Ce n'est pas la peine qu'elle réponde, que répondrait-elle ? Elle attend. Alors il lui demande : mais d'où venez-vous ? Elle dit qu'elle est la fille de l'institutrice de l'école des filles (...).

(G. Sand, Mare au Diable, XV, avec le récit de Petit-Pierre)

Pour ce qui concerne plus particulièrement $o r$, qui nous occupera désormais dans les pages qui suivent, l'avis de Ollier $(1995,23)$ revient à alléguer que «cette situation pose un problème intéressant de répartition : si la conjonction (mais, que / car, et) marque déjà l'existence d'un lien chaque fois spécifique entre E1 et E2, la présence de or n'en impose pas moins de mettre au jour une opération de rupture »? D'autre part, «or construit un seuil, qui se manifeste (...) par une discontinuité temporelle » (id., 26). A ce sujet, on notera que Sakari (1997), posant ainsi le problème de savoir dans quels termes délimiter la temporalité effective du marqueur (355-356), cite les deux exemples suivants :

Or pleust a Dieu que je sceus tout

De drois et de theologie !

(Bernard, 1782-1783)

Hé Dieu ! quel marchant!

Pleust or a Dieu qui n'y vist goutte ! 
L'auteure, justement, renvoie au fait qu' «on peut se demander si or placé après le verbe (...) a effectivement une valeur plutôt temporelle ». Cela étant, même si l'on imagine volontiers, dans cette distinction graphique, un «caprice orthographique»(358), la variante or et ore(s) a de quoi servir d'argument, bien que le critère positionnel ne soit pas toujours déterminant. Suivant, en effet, que le marqueur se place en position préverbale ou postverbale, la paragoge peut avoir eu pour objet d'orthographier le morphème en vue d'en expliciter non pas une acception particulière à vrai dire, mais bien le degré d'instantanéité exprimée. D'un autre côté, on notera que cette variabilité graphique et positionnelle du marqueur se révèle assez lisible dans le classement que dresse Buridant (2000) du or employé «en tant qu'opérateur de rupture énonciative d'une proposition P2, (quand il) marque un changement dans l'ordre des choses représenté par la proposition P1 antécédente » (519-521). D'autant que « cette rupture peut se présenter dans plusieurs schémas discursifs », enchérit-il (519), à savoir « au début d'une nouvelle réplique » (519-520), " au sein d'une même réplique » (520), " après un récit » (id.), ou « dans le cours d'un récit» (520-521). Pourtant, que or soit placé en début de syntagme verbal, voire, à l'occasion, suite à l'apostrophe, non seulement n'influence pas son éventuelle conjonctionnalité, mais encore le ramène dans bien des cas au to de l'énonciation, souvent paraphrasé par un là, tout de suite singulatif, dans un lien d'extériorité par rapport à un avant et un après discursifs :

Theophile

Or sui je venuz trop matin?

(Mir. Théophile, 204)

Pincedés

Ceste est bien au moy de le tieue.

Rasoirs

Et ceste, se g'i seuc lignier.

Li tavreniers

Segneur, or doi je apongnier?

Mais mout bien nous en convenra.

(St Nicolas, 1072-1075)

Qu'on leur attribue telle valeur temporelle ou telle autre, les deux occurrences de or placées en italiques ci-dessus ressortissent simultanément à un même instantané singulier. Intersegmentaux d'une réplique à l'autre, et donc interphrastiques ici, ceux-ci permettent au locuteur de revenir sur soi, en rupture hic et nunc avec la réplique de l'interlocuteur qui vient d'intervenir (Torterat, 2004). En outre, les deux questions concernées, qui se présentent comme de véritables objections, impliquent avec le marqueur une portée adversative, voire ouvertement dénégative par rapport au dit précédent, et insistent, en un sens, au moins autant sur la présence du locuteur qui intervient que sur l'intervention elle-même, en excluant toute forme d'itération. Il y a bien coordination, mais avec une rupture caractéristique de or, qu'une retranscription par donc ou par alors oblitèrera en partie. Quant à la place de l'opérateur dans le syntagme, celle-ci reste conforme à celle des coordonnants intersegmentaux en général, en position préverbale. En revanche, on est en droit de s'interroger sur le fait de savoir si nous avons là un automatisme véritablement contraint. A ce propos justement, voyons cette autre question :

Tuit cil de Rome haltement s'escrierent.

Dist l'apostoiles : «Que fais tu or, sainz Pere ? (...)»

(Cour. Louis, 1085-1086)

La place occupée par or, dans le SV et par rapport au SN en apostrophe, permet-elle, en l'occurrence, de le présumer comme concurrent de donc, dans son rôle d'incitation, tout en lui conjoignant l'acception d'à présent, tout de suite? Il existe bien sûr, au milieu d'une certaine variété d'emplois au regard des 
segments cotextuels dont or est entouré, des récurrences. Nous assistons en effet, lors des premiers classements à caractère statistique, à une présence accrue de or à la place 1 du segment phrastique, et cela dans le cadre d'une coordination interpropositionnelle, alors que l'opérateur ne semble apparaître qu'exceptionnellement en tournures réduplicatives, incidentes - ou les deux à la fois. L'injonction, quant à elle, est plus contraignante à cet égard, tandis que la question apparaît plus en marge.

Dans l'exemple du Couronnement de Louis, or marquera plusieurs opérations simultanément. En toute vraisemblance, il conserve sa primitive temporelle de marqueur du présent interlocutif, que nous pourrions d'ailleurs retranscrire par là ( Que fais-tu là ? ») - si nous ne tenions compte que d'elle - ou bien, de manière plus descriptive, en (tout) de suite, voire à présent / présentement, conformes à l'invariant de semelfactivité. Par ce biais, or constitue un appui (re)marqué à l'injonction ad hominem (et ici ad Deum), en suggérant au destinataire du propos combien la réponse à la question doit être prise pour une contrainte irrémissible, au sens plus général du terme.

Dans l'extrait cité, le pape exige en effet - ou feint d'exiger - une réponse quasi-simultanée, ce qui, ici, prend un coloris ouvertement oratoire, de même qu'un caractère presque politique. L'énonciateur pose une question, mais il le fait ostensiblement, là où un tel souci d'exhibition s'expliquera bien entendu par la teinte cérémoniale, et en tous les cas publique, de cet appel. Pour cela, le pape en fera même peut-être un peu trop, car son propos quasi-mosaïque devient, à la suite de ce que nous avons lu, directement comminatoire :
Se il i muert, c'iert mal destinee.
En ton mostier n'iert mais messe chantee,
Tant com je vive ne que j'aie duree.

(1087-1089)

A l'occasion de ce type d'emploi, où or prend, dans le contexte, une dimension quasiment injonctive, celui-ci pourra se retranscrire par donc sans que sa dimension temporelle soit atteinte au point de se voir écartée en tant que telle. Par ailleurs, on note ici et là, dans le discours direct, des collocations dans lesquelles donc, sans enchérir sur or, vient à proprement parler en surdétermination de ce dernier, afin d'insister sur le caractère à la fois pressant, instantané et singulier de la question posée. Au demeurant, dans le cas donc où or serait postposé par rapport au SV dans son ensemble, la coordination opérée avec un segment précédent ne paraît pas envisageable, même si, à certains moments, il semble que l'opérateur effectue bien la transition avec un avant-texte particulier. A ce propos d'ailleurs, Ollier (op. cit., 27) indique qu'en position postverbale, or a plus exactement pour rôle «d'établir (le procès) à proximité de to » (autrement dit le présent de l'énonciateur), ce qui nous renvoie encore une fois à la temporalité marquée de l'opérateur (dans ce cas un adverbe), plus que sur son caractère proprement conjonctionnel au titre de la coordination grammaticale.

\section{Quelques éléments de corroboration}

Pour ce qui relève donc de or, le bas-latin périphrastique HA(C) HORA*, qui connaîtra sans doute une influence de ILLA HORA*, désigne proprement «cette heure », et de manière plus étendue «ce momentlà », «cet instant-là ». D'où la forme d'ore, qui se maintient jusqu'au XVII ${ }^{\mathrm{e}}$ siècle, pour se teinter peu à peu d'archaïsme et se fixer aux branches de quelques locutions. Un remarquable exposé critique en est établi dans le Dictionnaire historique de Rey (dir., 1992 : 1374), qui n'oublie pas ses emplois interjectifs, mais le problème demeure encore une fois cette stabilisation du marqueur dans lesdites valeurs plus exactement argumentatives, où il se grammaticalise par restriction d'acceptions (et non de sens).

Sur ce propos, Dubois (1997), qui revient plus généralement, concernant la construction d'invariants, sur la «stabilisation par décontextualisation, et, du même coup, le figement par élimination de la temporalité » (108), nous invite à envisager ce processus en termes de représentation : 
(...) la construction de la classe paradigmatique va effacer la temporalité dans la représentation cognitive de l'objet, et constituer une forme de figement dans son invariance, sa permanence cognitive indépendante du temps de l'action ou de la situation qui a permis de l'identifier comme tel. (même page)

Dans le cadre d'un relevé statistique pour l'ancien et le moyen français, le fait que ce soit à l'assertion et l'injonction que revienne le plus grand nombre d'emplois de or apporte une indication complémentaire non négligeable, alors qu'en revanche, on notera l'incompatibilité quasi-générale de or et de la question. Pour les cas du Charroi de Nismes, de St Nicolas et du Palatinus, un tel relevé donnera les proportions suivantes $^{10}$ :

\begin{tabular}{|l|c|c|c|}
\hline & Charroi de Nismes & Saint Nicolas & Palatinus \\
\hline $\begin{array}{l}\text { Emplois autodéterminés } \\
\text { (généralement interjectifs et } \\
\text { locutionnels) }\end{array}$ & $0 \%$ & $20 \%^{11}$ & $18 \%^{12}$ \\
\hline $\begin{array}{l}\text { Emplois dans l'assertion } \\
\text { (antéposés), }\end{array}$ & $52 \%^{13}$ & $30 \%^{14}$ & $36 \%^{15}$ \\
\hline dans la question, & $0 \%$ & $2 \%^{16}$ & $0 \%$ \\
\hline dans l'injonction & $17 \%{ }^{17}$ & $46 \%^{18}$ & $39 \%{ }^{19}$ \\
\hline Cas de postpositions & $31 \%{ }^{20}$ & $2 \%^{21}$ & $7 \%{ }^{22}$ \\
\hline
\end{tabular}

Les emplois proprement interjectifs de or (ou plutôt assimilés comme tels), locutionnels de manière générale, apparaissent ainsi tournés vers une concrétisation dialogale de l'énoncé, et un schéma plus exactement interlocutif, ce qui confirme notamment le fait que or fonde surtout sa dimension temporelle dans une instantanéité non reconduite. Mais le contexte est là aussi pour le moins significatif, même si la présence d'un co-énonciateur dans la motivation des emplois du marqueur ne lui est pas entièrement extérieure. Dans la question par exemple, or indique une forme d'exigence de dicto, et que celle-ci soit comprise ainsi ou ne soit pas saisie comme telle par son destinataire ressortit, notamment, à la compréhension de l'autre dans l'instant. Dans l'injonction en revanche, cette acception du marqueur s'avère transparente, car elle se fonde dans l'évident caractère semelfactif de ce qui est prédiqué. Par ailleurs, les cas de postpositions apparaissant de moins en moins en diachronie, la position préverbale de or va conforter plus avant sa matérialité de conjonction singulative.

Il conviendrait d'établir un relevé similaire pour (a)lors, à l'appui des concordances qui sont à notre disposition. Il y aurait ainsi de bonnes chances pour que la semelfactivité représentée conforte non seulement les caractéristiques catégorielles de conjonction pour or et d'adverbe pour (a)lors sur le plan phrastique, mais aussi le rôle de cohéreurs textuels des deux opérateurs, lesquels indiquent souvent les moments singuliers du texte en lien avec des instantanés du discours. Quoi qu'il en soit, ces faits témoignent de la capacité, pour des opérateurs conjonctifs et adverbiaux, de marquer une semelfactivité non verbale sans doute insuffisamment désignée comme telle en linguistique française.

\section{Références}

Achard, P. (1992). « Entre deixis et anaphore : le renvoi du contexte en situation. Les opérateurs «alors » et «maintenant» en français », in Mary-Annick Morel et Laurent Danon-Boileau (éds), La Deixis, Paris, PUF, 583-592. 
Antoine, G. (1962). La Coordination en français, I-II, Paris, d'Artrey.

Authier-Revuz, J. (1991). «Hétérogénéités et Ruptures. Quelques repères dans le champ énonciatif», in Herman Parret (éd), Le Sens et ses Hétérogénéités, C.N.R.S. éditions, 139-151.

Badiou-Monferran, C. (2000). Les Conjonctions de coordination, ou «l'art de lier ses pensées » chez La Bruyère, Paris, Champion.

Badiou-Monferran, C. (2003). «Quelques Aspects de la concurrence des graphies ore, ores et or au début du XVIIe siècle : distribution sémiologique et recomposition du système des connecteurs », Français moderne LXXI, 2, 211-247.

Benazzo, S. (2000). L'Acquisition de particules de portée en français, anglais et allemand en L2. Etudes longitudinales comparées, thèse de doctorat, Université Paris 8 / Freie Universität Berlin.

Blumenthal, P. (1980). La Syntaxe du message, Tubingen, Niemeyer, Max Verlag (180).

Bouchard, R. (2001). "Alors, donc, mais..., "particules énonciatives» et/ou «connecteurs»? », Syntaxe et Sémantique 3, Crisco, Caen, PUC, 63-73.

Bougy, C. (2000). "Les Connecteurs temporels et l'Apparition de lors que dans la langue française », Syntaxe et Sémantique 1, Caen, PUC, 39-78.

Bres, J. dir. (1999). L'Imparfait dit narratif, numéro des Cahiers de Praxématique, 32, Montpellier 3, Praxiling.

Buridant, C. (2000). Grammaire nouvelle de l'ancien français, Paris, Sédes.

Buvet, P.A., Girardin, Ch., Gross, G., Groud, C. (2005). «Les Prédicats d'affect », Lidil, 32 (version électronique à l'url : http://lidil.revues.org/document104.html ).

Dik, S.C. (1997). The Theory of functional Grammar, Berlin, Mouton de Gruyter.

Dubois, D. (1997). «Catégories, Prototypes et Figements. Constructions d'invariants et systèmes symboliques », in Michel Martins-Baltar (éd.), La Locution, entre langue et usage, Fontenay-Saint Cloud, ENS éditions, 103-129.

Feuillet, J. (1990). «Place de la coordination », L'Information grammaticale 46, 4-7.

Franckel, J. J. (1989). « Alors », in Etudes de quelques marqueurs aspectuels du français, Genève-Paris, Droz, 355368 (cet article reprend en partie Franckel, 1987 : «Alors - alors que », paru dans BULAG 13, Besançon, 17 49).

Gerecht, M.-J. (1987). «Alors : opérateur temporel, connecteur argumentatif et marqueur de discours », Cahiers de Linguistique française 8, 69-79.

Jayez, J. (1988). «Alors : description et paramètres », Cahiers de Linguistique française 9, 135-175.

Krifka, M. et al. (1995). «Genericity : an introduction », in Greg Carlson et Francis Jeffry Pelletier, The Generik Book, Chicago, CUP, 1-124.

Laca B. (2002). Temps et aspect. De la morphologie à l'interprétation, Saint-Denis, PUV.

Lefeuvre, F., Le Goffic, P. (1999). La Phrase averbale en français, Paris, L'Harmattan.

Mantcheva, A. (2004). Oeuvres de K. Mantchev. La Linguistique, Paris, L'Harmattan.

Ollier, M.-L. (1995). «Or, opérateur de rupture », Linx 32, 13-31.

Portine, H. (2001). «Connecteurs et marqueurs : quels termes métalinguistiques ? , in Bernard Colombat et Marie Savelli (éds), Métalangage et Terminologie linguistique, Orbis supplementa 17, Louvain, Peeters, 239-249.

Ruppli, M. (1988). La Coordination en français moderne. Théorie à l'épreuve d'un corpus., Paris III, thèse de doctorat nouveau régime.

Sakari, E. (1997). «Observations sur quelques adverbes de temps (or, lors, alors) en moyen français », in Bernard Combettes et Simone Monsonégo (éds), Le Moyen Français, 351-369.

Smith, S. (1991). The Parameter of Aspect, Dordrecht, Kluwer.

Stoll, S. (1998). « The Role of Aktionsart in the acquisition of Russian aspect », First Language 18, 351-378. 
Torterat, F. (2004). «Si et $\mathrm{Or}$ comme cohéreurs (para)textuels en ancien et en moyen français », in Olivier Soutet et Danièle James-Raoul (éds), Par les Mots et les Textes..., Mélanges de langue, de littérature et d'histoire des sciences médiévales offerts à Claude Thomasset, Presses de l’Université Paris-Sorbonne, 757-773.

Vion, R. (1995). «La Gestion pluridimensionnelle du dialogue », Cahiers de Linguistique française 17, Genève, 179-203.

Wauquier-Gravelines, S. (1999). "Segmentation lexicale de la parole continue. La Linéarité en question», Recherches Linguistiques de Vincennes 28, 133-156.

1 On rappellera à cet égard le programme de recherche conduit par P. Cabredo Hofherr et B. Laca dans le contexte de l'unité FR 2559, sur les pluralités nominale et verbale, et qui porte notamment sur les marqueurs ad-verbaux de semelfactivité ainsi que sur les adverbes impliqués dans cette problématique.

2 Cf. : Et, au moment où, vraiment, on n'allait plus pouvoir supporter d'être là, où l'on allait crier : «Alors, qu'est-ce que vous faites ? », il y eut une grosse détonation et l'homme tomba. (J. Giono, Un Roi sans divertissement, LP, p. 88)

3 On remarquera ici que le même constat s'applique à $o r$.

$4 \quad$ Vion $(1995,184)$ nous explique qu' « en abordant les places discursives, nous descendons d'un cran dans l'analyse (par rapport au cadre social), puisque l'ordre généralement concerné par ce type de phénomène est la séquence. La séquence se présente comme un moment interactionnel organisé autour d'une même fonctionnalité. On a pu parler de séquence d'ouverture, de clôture, de séquence latérale pour gérer un problème de communication ou obtenir un certain nombre d'informations nécessaires à la poursuite de l'échange. Nous parlerons également de séquence, dans le sens de J.M. Adam, moins pour y positionner des types textuels que des tâches cognitivo-discursives ». " Deux problèmes se posent dès lors qu'on envisage (la représentation prosodique qui regroupe les éléments lexicaux concernés) : quelles sont la taille et la nature de l'unité prosodique permettant l'accès au lexique ? », questionne pour sa part Wauquier-Gravelines $(1999,146)$.

5 «La reprise opérée par alors doit correspondre en même temps à un décrochage, insiste Franckel (368), entre les repères SITi et SITj. En l'absence de tout décrochage, le fonctionnement de alors est bloqué. C'est ce qui se produit dans l'exemple suivant relevé par J. Jayez (1981) : Pierre, vous savez, celui qui... Pierre alors, vous attend. J. Jayez tire argument de cet exemple pour mettre en doute et même rejeter l'idée selon laquelle alors marquerait une reprise, alors même que, selon nous, il la conforte. Notons à ce propos que la notion de reprise affleure nettement dans la glose la plus couramment associée à alors : à ce moment-là. Dans cette glose, apparaît d'une part ce, trace d'une identification, et d'autre part là comme délimitation d'un espace, comme démarcation d'un espace ouvert (tel que le construirait $\mathrm{ici}$ ). Quant au terme de moment, il ne faut pas lui attribuer une acception spécifiquement temporelle, mais le comprendre dans le sens plus général de repère. Le fait d'avoir P en SITi permet de prédiquer Q de SITj, reprise de SITi ».

6 La problématique de l'événementialité, que la linguistique s'est appropriée notamment à travers les contributions de Vendler, mais aussi Comrie et Dowty, permet, par son caractère généraliste, de rejoindre les approches philosophiques de la semelfactivité à l'appui de Lévinas et de Jankélévitch, avec des reformulations envisageables, en termes d'unicité, qui ont fait plusieurs fois la démonstration de leur opportunité.

$7 \quad$ Cf. et or et mais or (24-26)

$8 \quad$ Dans les cas cités, si nous retranscririons volontiers le premier syntagme verbal avec or par " plût à Dieu que.. ", la postpositon de l'opérateur, dans le deuxième exemple, nous incite à le retranscrire dans une proximité plus effective par rapport au to de l'énonciation, autrement dit en «Plaise à Dieu que.. ». 
Un défaut, heureusement secondaire chez ces auteurs, se remarque en partie chez J.J. Franckel et chez J. Jayez vis-à-vis d'alors, notamment quand ces derniers sollicitent un appareil classificatoire culiolien qui vient d'ailleurs servir d'intermédiaire dans leurs contributions d'ensemble, quand bien même il s'agirait de convenir du marquage d'une « situation nouvelle ».

10 En voici les références éditoriales : le Charroi de Nismes, éd. Jean Louis Perrier, Paris, 1968 (Champion) ; Bodel Jehan, Jeu de Saint Nicolas, éd. Albert Henry, Genève, 1981 (Droz) ; La Passion du Palatinus, éd. Grace Frank, Paris, 1922 (Champion).

11 224-298-580-668-722-1326-1412-1500 (or cha) ; 696-1002-1027-1048-1496 (or tost) ; 1282 (or sus) ; 1360-1533 (or jus) ; 1322 (or hors). Avec un or-vocatif en 566.

12 237-247-271-363-740 (or ça) ; 1274-1442-1555-(bis)1784 (or tost) ; (bis)35-121-787-1235(ter) 1724 (or sus) ; 1552 (or dont).

295 (avec post. sujet)-301 (id.)-340-382-431-592-615 (id.)-892-895-1191 (avec si)-1356-1463.

$14 \quad 132-138-205-238-394 \quad$ (did.)-453 (did.)-468-573-697-806-903-925-1051-1119-1124-1138-1182-1257-1340$1341-1536$.

15 59-85-246-379-476-539-627-630-633-645-652 (ore)-769 (id.)-808-812-821-833-835-982-10241037-1060-1084-1089-1106(ore)-1108-1142-1155-1248-1260-1269-1297-1418-1438-1449-1586-16451943-1947.

161074.

17 286-583 (avec dont)-1069 (avec donques)-1162.

18 114-220-296 (avec dont)-396 (avec infin.)-399-459-510-517 (or me di)-578-594-624-686-736778-814 (avec dont)-942 (id.)-945-960-1021 (id.)-1098-1132-1156 (avec infin.)-1184-1228 (avec infin.)1333-1353-1362-1382 (avec infin.)-1425 (or me di).

19 123-129 (or - savoir)-196-273-315 (ore)-333-397-409-438-454-475-499-565-636-645-651691-698-764-839-855-866-894-1007-1010-1027-1045-1047-1278-1324-1385-1489-1538 (or - savoir)1577 (or - dire)-1676-1678-1751 (avec dont)-1753-1771 (or - savoir à la troisième personne)-19501966-1989.

20 252-410-1013-1034 (dans des or antéposé)-1132-1250 (id.)-1329. Avec, pour inf., les ore dans St Nicolas (278-449-745-773-820-849-1109-1192-1277-1347-1386-1388-1411-1523), et dans le Charroi (400-403-534-537-799-821-923-940-1022-1220-1319-1336-1341-1345), où le ore de 593 correspond à or injonctif.

21

690.

22 359-505-1100-1189-1248-1289-1533. 